\title{
Some Kinds of Sheaf Control Problems for Control Systems
}

\author{
Nguyen Dinh Phu*, Phan Van Tri \\ Faculty of Mathematics and Computer Science, Vietnam National University, Ho Chi Minh City, Vietnam \\ Email: *ndphu_dhtn@yahoo.com.vn
}

Received November 8, 2011; revised December 20, 2011; accepted December 27, 2011

\begin{abstract}
Recently, the field of differential equations has been studying in a very abstract method. Instead of considering the behaviour of one solution of a differential equation, one studies its sheaf-solutions in many kinds of properties, for example, the problems of existence, comparison, ... of sheaf solutions. In this paper we study some of the problems of controllability for sheaf solutions of control systems.
\end{abstract}

Keywords: Control Systems; Sheaf Solutions; Control Theory

\section{Introduction}

In [1-4] the authors have investigated sheaf solutions of control differential equations in the fields: comparison of sheaf solutions in the cases' two admissible controls $u(t)$ and $\bar{u}(t)$, and some initial conditions $x_{0} \in H_{0}$, $\bar{x}_{0} \in \bar{H}_{0}$, where the Hausdorff distance between the sets of initials $H_{0}$ and $\bar{H}_{0}$ is enough small.

The problems of sheaf controllability and sheaf optimization are still open. The present paper is organized as follows. In Section 2, we review some facts about sheaf solutions. In Section 3 we give many kinds of sheaf control problems, of sheaf controllability optimal problems.

\section{Preliminaries}

In $n$-dimension Euclidian space $R^{n}$ usually we have considered the control systems (CS):

$$
\frac{\mathrm{d} x(t)}{\mathrm{d} t}=f(t, x(t), u(t))
$$

where $x:\left[t_{0}, T\right] \rightarrow R^{n}, u(t) \in U \subset R^{d}$

$f:\left[t_{0}, T\right] \times R^{n} \times R^{d} \rightarrow R^{n}$. A solution to CS (2.1) is represented by:

$$
x(t)=x\left(t_{0}, x_{0}, t, u\right)=x_{0}+\int_{t_{0}}^{t} f(s, x(s), u(s)) \mathrm{d} s
$$

$x_{0} \in H_{0} \subset R^{n}, t \in\left[t_{0}, T\right] \subset R^{+}, H_{0}$ is a collection of some given initials.

Definition 2.1. We say that a control $u(t) \in U$ is admissible, if:

1) $u(t)$ satisfies (2.2) for all $t \geq t_{0}$;

\footnotetext{
${ }^{*}$ Corresponding author.
}

2) $u(t)$ is bounded by norm $\|u(t)\|$.

That means the functions $u(t)$ are measurable (integrable) satisfiying almost everywhere on $\left[t_{0}, T\right]$ the relationships (2.1) and (2.2), then $x(t)$ is called the trajectory of the CS (2.1) and $u(t)$ is called the control. Therefore, we shall always understand a pair of functions $(x(t), u(t))$ interrelated by the relationship (2.1) and (2.2). It is clear that several controls $u(t)$ can correspond to one $x(t)$ trajectory and if CS (2.1) has a nonunique solution, then several trajectory $x(t)$ can correspond to one control $u(t)$.

Definition 2.2. A state pair $\left(x\left(t_{0}\right), x\left(t_{1}\right)\right)$ of solutions of control systems (2.1) will be a controllable if after time $t_{1}$ we shall find a control $u(t) \in U$ such that:

$$
x\left(t_{1}\right)=x\left(t_{0}, t_{1}, u\left(t_{1}\right)\right)=x_{1}
$$

Definition 2.3. A control system (2.1) is said to be:

(GC) global controllable if every state pair of set solution $x\left(t_{0}\right), x\left(t_{1}\right) \in R^{n}$.

(GA) global achievable if for every $x_{1} \in R^{n}$ we have a state pair of solutions $0, x_{1}$ that is GC.

(GAZ) global achievable to zero if for every $x_{1} \in R^{n}$ we have a state pair $x_{1}, 0 \in R^{n}$ that will be controlable.

In [2] the authors have compared the sheaf solutions for set control differential equations (SCDEs).

In [4] the author has study the problems (GC), (GA) and (GAZ) for set control differential equations (SCDEs).

$$
u(t), \bar{u}(t) \in U \subset R^{p}, t \in[0, T] .
$$

Definition 2.4. A sheaf solution (or sheaf trajectory) $H_{t, u}$ is denoted by a number of solutions that make into 
sheaves (lung one on top of the other and often tied together) for all $t \in\left[t_{0}, T\right]$ :

$$
H_{t, u}=\left\{x\left(t_{0}, x_{0}, t, u(t)\right) \mid x_{0} \in H_{0}, t \in\left[t_{0}, T\right], u(t) \in U\right\}
$$

Definition 2.5. A cut-set (a cross-area) of sheaf solution $H_{t, u}$ at time $t_{1}$ is denoted by:

$$
\begin{aligned}
& H_{t_{1}, u\left(t_{1}\right)}=\left\{x\left(t_{1}\right)=x\left(t_{0}, x_{0}, t_{1}, u\left(t_{1}\right)\right) \mid x_{0} \in H_{0}\right\}, \\
& t_{1} \in\left[t_{0}, T\right], u\left(t_{1}\right) \in U
\end{aligned}
$$

\section{Main Results}

Let's consider again the control systems (CS):

$$
\frac{\mathrm{d} x(t)}{\mathrm{d} t}=f(t, x(t), u(t))
$$

where $t \in\left[t_{0}, T\right] \subset R^{+}, \quad x(t) \in Q \subset R^{n}, Q$ is a compact set in $R^{n}$ and $u(t) \in U \subset R^{d}$-admissible controls. Assume that for CS (3.1) there exists solution (2.2) and sheaf solution (2.4).

We will need the following hypotheses on the data of control problem for CS (3.1):

(Hf1):

$$
\begin{aligned}
& \|f((t, \bar{x}(t), u(t))-f(t, x(t), u(t)))\| \\
& \leq c(t)(1+\|\bar{x}(t)-x(t)\|) \\
& \|f((t, \bar{x}(t), \bar{u}(t))-f(t, x(t), u(t)))\| \\
& \leq c_{2}(t)(1+\|\bar{x}(t)-x(t)\|+\|\bar{u}(t)-u(t)\|)
\end{aligned}
$$

(Hf2):

where $c_{1}(t), c_{2}(t) \in L([0, T])$.

Assume that at all $t \in I=[0, T], u\left(t_{0}\right)=0, x\left(t_{0}\right)=x_{0}$ for two admissible controls $u(t), \bar{u}(t) \in U$ we have two forms of sheaf solutions:

$$
\begin{aligned}
& H_{t, u}=\left\{x(t)=x\left(t_{0}, x_{0}, t, u(t)\right) \mid x_{0} \in H_{0}, t \in I, u(t) \in U\right\} \\
& H_{t, \bar{u}}=\left\{\bar{x}(t)=x\left(t_{0}, x_{0}, t, \bar{u}(t)\right) \mid x_{0} \in H_{0}, t \in I, \bar{u}(t) \in U\right\}
\end{aligned}
$$

where $x(t)=x\left(t_{0}, x_{0}, t, u(t)\right)$-solution of CS (2.1) (see Figure 1).

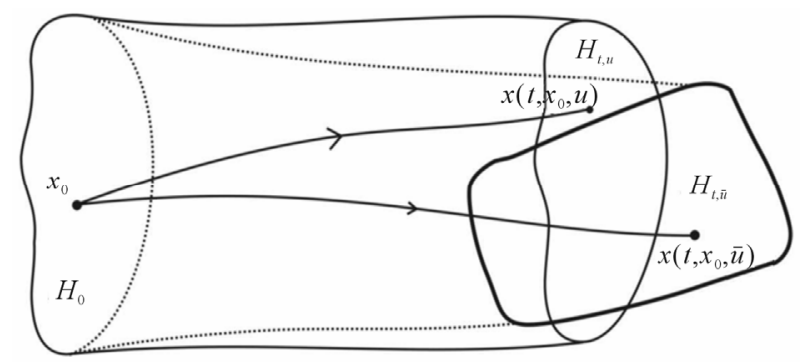

Figure 1. The sheaf solutions of CS (2.1) in two admissible controls.
Definition 3.1. The Hausdorff distance between set $H_{t, u}$ and $H_{t, \bar{u}}$ is denoted by:

$$
\begin{aligned}
& d_{H}\left[H_{t, u}, H_{t, \bar{u}}\right] \\
& =\max \left\{\sup _{x(t) \in H_{t, u}} d\left(x(t), H_{t, \bar{u}}\right), \sup _{\bar{x}(t) \in H_{t, \bar{u}}} d\left(\bar{x}(t), H_{t, u}\right)\right\}
\end{aligned}
$$

Definition 3.2. The pair of the any sets $H_{0}, H_{1} \subset R^{n}$ will be controllable if after time $t_{1}$ we shall find a control $u(t) \in U$ and one map $\sigma: R^{n} \times R \rightarrow R^{n}$ such that:

$$
\sigma\left(H_{0}, t_{1}\right)=H_{1}
$$

Theorem 3.1. Under Hypothes (Hf1), let $x_{0} \in H_{0} \subset R^{n}$ -is initial, any set $H_{1} \subset R^{n}$. The pair of the sets $H_{0}, H_{1} \subset R^{n}$ will be controllable if:

1) $x(t)$ belongs to solutions of CS (3.1), and

2) $H_{1}$ is cut-set of sheaf solution to (3.1), that means

$$
H_{1}=\left\{x\left(t_{0}, x_{0}, t_{1}, u\left(t_{1}\right)\right) \mid x_{0} \in H_{0}, u(t) \in U, t_{1} \in I\right\} .
$$

Proof. If $x(t)$ belongs to solutions of CS(3.1) then it is

$$
x(t)=x\left(t_{0}, x_{0}, t, u\right)=x_{0}+\int_{t_{0}}^{t} f(s, x(s), u(s)) \mathrm{d} s .
$$

For any $x_{1} \in H_{0}$ we have a pair $\left(x_{0}, x_{1}\right)$ that is controllable, because $x_{1}=x\left(t_{0}, x_{0}, t_{1}, u\left(t_{1}\right)\right)$, where $x_{1} \in H_{1}$ - cut-set of sheaf solutions with

$$
H_{1}=\left\{x\left(t_{0}, x_{0}, t_{1}, u\left(t_{1}\right)\right) \mid x_{0} \in H_{0}, u(t) \in U, t_{1} \in I\right\}
$$

As in results, we have one map moving $H_{0}$ to $H_{1}$ that means $\sigma\left(H_{0}, u\left(t_{1}\right)\right)=H_{1}$.

Definition 3.3. The control system (3.1) is said to be:

(SC1) sheaf controllable in type 1, if for all $\epsilon>0$, there exists $\delta_{1}(\epsilon)$ and admissible controls $u(t), \bar{u}(t)$ that satisfy $\|\bar{u}(t)-u(t)\| \leq \delta_{1}$ then

$$
d_{H}\left[H_{t, u(t)}, H_{t, \bar{u}(t)}\right]<\epsilon .
$$

(SC2) sheaf controllable in type 2 for any admissible control $u(t)$, if for all $\epsilon>0$, there exists $\delta_{2}(\epsilon)>0$ such that the initials $x_{0} \in H_{0}, \quad \bar{x}_{0} \in \bar{H}_{0}$ with $d_{H}\left(H_{0}, \bar{H}_{0}\right)<\delta_{2}$ then

$$
d_{H}\left[H_{t, u}, \bar{H}_{t, u}\right]<\epsilon .
$$

(SC3) sheaf controllable in type 3 , if for all $\epsilon>0$, there exist $\delta_{1}(\epsilon), \quad \delta_{2}(\epsilon)>0$ such that the initials $x_{0} \in H_{0}, \quad \bar{x}_{0} \in \bar{H}_{0}$, with $d_{H}\left[H_{0}, \bar{H}_{0}\right]<\delta_{2}$ and for any admissible controls $u(t), \bar{u}(t)$ that satisfy $\|\bar{u}(t)-u(t)\| \leq \delta_{1}$ then

$$
d_{H}\left[H_{t, u}, \bar{H}_{t, \bar{u}}\right]<\epsilon \text { or } d_{H}\left[H_{t, \bar{u}}, \bar{H}_{t, u}\right]<\epsilon .
$$

Lemma 3.1. Under Hypothes (Hf1), for all $\epsilon>0$, 
there exists $\delta(\epsilon)>0$ if control system (3.1) with:

$$
\|\bar{x}(t)-x(t)\| \leq \delta
$$

then two cut -sets of sheaf-solutions of CS (3.1) satisfy an estimate:

$$
d_{H}\left[H_{t_{1}, u\left(t_{1}\right)}, H_{t_{2}, u\left(t_{2}\right)}\right]<\epsilon
$$

Proof. Suppose that for CS (3.1) the right hand side $f(t, x(t), u(t))$ satisfies (Hf1) then there exists unique solution $x(t)=x\left(t_{0}, x_{0}, t, u(t)\right)$ which satisfies (2.2).

If $H_{t, u}$-sheaf solution of CS (3.1) then for admissible control $u(t) \in U \subset R^{d}$ we have the cut-sets at any times $t_{1}, t_{2}$, that satisfy estimate (3.7):

$$
d_{H}\left[H_{t_{1}, u\left(t_{1}\right)}, H_{0}\right]<\frac{\epsilon}{2} \text { and } d_{H}\left[H_{t_{2}, u\left(t_{2}\right)}, H_{0}\right]<\frac{\epsilon}{2} .
$$

We have

$$
\begin{aligned}
& d_{H}\left[H_{t_{1}, u\left(t_{1}\right)}, H_{t_{2}, u\left(t_{2}\right)}\right] \\
& \leq d_{H}\left[H_{t_{1}, u\left(t_{1}\right)}, H_{0}\right]+d_{H}\left[H_{t_{2}, u\left(t_{2}\right)}, H_{0}\right]<\epsilon .
\end{aligned}
$$

Theorem 3.2. Assume that, under Hypothes (Hf2), the admissible controls $u(t), \bar{u}(t)$ that satisfy $\|\bar{u}(t)-u(t)\| \leq \delta_{1}$, then CS (3.1) is sheaf-controllable SC1.

Proof. Suppose that for CS (3.1) the right hand side $f(t, x(t), u(t))$ satisfies (Hf2) then there exists unique solution $x(t)=x\left(t_{0}, x_{0}, t, u(t)\right)$ which satisfies (2.2).

If $H_{t, u}$-sheaf solution of CS (3.1) then for admissible control $u(t) \in U \subset R^{d}$ we have the cut-sets at every times $t$, that satisfy estimate (3.7): $d_{H}\left[H_{t, u}, H_{0}\right]<\frac{\epsilon}{2}$ and $d_{H}\left[H_{t, \bar{u}}, H_{0}\right]<\frac{\epsilon}{2}$.

We have

$$
\begin{aligned}
& d_{H}\left[H_{t, u(t)}, H_{t, \bar{u}(t)}\right] \\
& \leq d_{H}\left[H_{t, u(t)}, H_{0}\right]+d_{H}\left[H_{t, \bar{u}(t)}, H_{0}\right]<\epsilon,
\end{aligned}
$$

as results the CS (3.1) is sheaf-controllable SC1. (口)

Corollary 3.1. If CS (3.1) is SC1, the right hand side $f(t, x(t), u(t))$ satisfies condition of lemma 3.1 then for all $\epsilon>0$ there exists $t_{1} \in[0, T]$ such that:

$$
\left|t_{1}-t_{0}\right|<\delta, d_{H}\left[H_{0}, H_{1}\right]<\epsilon
$$

Proof. Because solution of CS (3.1) is equivalent: $x(t)=x_{0}+\int_{t_{0}}^{t} f(s, x(s), u(s)) \mathrm{d} s$ then

$$
H_{1}=\left\{x\left(t_{0}, x_{0}, t_{1}, u\left(t_{1}\right)\right)\left|x_{0} \in H_{0},\right| t_{1}-t_{0} \mid<\delta, u(t) \in U\right\}
$$

by lemma 3.1 we have: $d_{H}\left[H_{0}, H_{1}\right]<\epsilon$

Theorem 3.3. Under hypothes (Hf1), assume that the initials $x_{0} \in H_{0}, \bar{x}_{0} \in \bar{H}_{0}$ for all $\epsilon>0$, there exists $\delta(\epsilon)>0$ such that: $d_{H}\left[H_{0}, \bar{H}_{0}\right]<\delta$ then for any admissible control $u(t)$ we have:

$$
d_{H}\left[H_{t, u}, \bar{H}_{t, u}\right]<\epsilon
$$

that means CS (3.1) is sheaf controllable CS2.

Proof. We have estimate

$$
\begin{aligned}
& d_{H}\left[H_{t, u}, \bar{H}_{t, u}\right] \\
& <d_{H}\left[H_{t, u}, H_{0}\right]+d_{H}\left[H_{0}, \bar{H}_{0}\right]+d_{H}\left[\bar{H}_{0}, \bar{H}_{t, u}\right]
\end{aligned}
$$

For all $\epsilon>0, \exists \delta(\varepsilon)$ such that $\forall t:\left|t-t_{0}\right|<\delta$ $d_{H}\left[H_{t, u}, H_{0}\right]<\delta, d_{H}\left(H_{0}, \bar{H}_{0}\right)<\delta$ and

$$
d_{H}\left[\bar{H}_{0}, \bar{H}_{t, u}\right]<\delta,
$$

choosing $\delta=\frac{\epsilon}{3}$ then we have $d_{H}\left[H_{t, u}, \bar{H}_{t, u}\right]<\epsilon$. As results imply that CS (3.1) is SC2.

Theorem 3.4. Under Hypothes (Hf2), assume that for all $\epsilon>0, \exists \delta(\epsilon)>0$ and satisfy the followings:

1) $u(t), \bar{u}(t) \in U,\|\Delta u\|<\delta$

2) $H_{0}, \bar{H}_{0} \subset Q: d_{H}\left[H_{0}, \bar{H}_{0}\right]<\delta$, then for any admissible controls $u(t), \bar{u}(t)$ we have:

$$
d_{H}\left[H_{t, u}, \bar{H}_{t, \bar{u}}\right]<\epsilon
$$

that means CS (3.1) is sheaf controllable CS3.

Proof. Beside (2.4) for $H_{0}$ and $\bar{H}_{0}$ we have:

$$
\begin{aligned}
& \bar{H}_{t, u}=\left\{x\left(t_{0}, \bar{x}_{0}, t, u(t)\right) \mid \bar{x}_{0} \in \bar{H}_{0}, u(t) \in U, t \in I\right\} \\
& \bar{H}_{t, \bar{u}}=\left\{x\left(t_{0}, \bar{x}_{0}, t, \bar{u}(t)\right) \mid \bar{x}_{0} \in \bar{H}_{0}, \bar{u}(t) \in U, t \in I\right\}
\end{aligned}
$$

and estimate $d_{H}\left[H_{t, u}, \bar{H}_{t, \bar{u}}\right]$ as following:

$$
\begin{aligned}
d_{H}\left[H_{t, u}, \bar{H}_{t, \bar{u}}\right] & <d_{H}\left[H_{t, u}, H_{0}\right]+d_{H}\left[H_{0}, \bar{H}_{0}\right] \\
& +d_{H}\left[\bar{H}_{0}, \bar{H}_{t, u}\right]+d_{H}\left[\bar{H}_{t, u}, \bar{H}_{t, \bar{u}}\right] .
\end{aligned}
$$

Choosing $\delta=\frac{\epsilon}{4}$, we have:

$$
d_{H}\left[H_{t, u}, \bar{H}_{t, \bar{u}}\right]<\epsilon
$$

Definition 3.4. We say that for control system (3.1) are given OCP - the optimization control problem if it denotes:

$$
\left\{\begin{array}{l}
\min _{u \in U} I(u) \\
\frac{\mathrm{d} x(t)}{\mathrm{d} t}=f(t, x(t), u(t)) \\
x_{0} \in H_{0} \subset R^{n}, u(t) \in U \subset R^{d}, t \in[0, T] \subset R^{+} \\
I(u)=\int_{0}^{T} \varphi(t, x(t), u(t)) \mathrm{d} t+h(x(T))
\end{array}\right.
$$

where $h(x(T))=V(T, x)$, such that $V(T, x)$ is solution 
to Hamillton Jacobi Bellman (HJB)—partial differential equation:

$$
\frac{\partial V(t, x)}{\partial t}+\left[\varphi(t, x, u)+\left\langle\frac{\partial V}{\partial x}, f(t, x, u)\right\rangle\right]=0
$$

We have to find the optimal control $u^{*}(t) \in U$ for OCP (3.8).

Lemma 3.1. In optimization control problems (3.8) if $V(T, x)=h(x(T))$ then $V\left(0, x_{0}\right) \leq I(u)$, $\forall u \in U \subset R^{d}$

Proof. Putting $\frac{\mathrm{d} y(t)}{\mathrm{d} t}=\frac{\partial V}{\partial t}+\left\langle\frac{\partial V}{\partial x}, f(t, x, u)\right\rangle$

we have integral for all $t \in[\alpha, \beta] \subset I=[0, T]$ :

$$
y(\beta)-y(\alpha)=\int_{\alpha}^{\beta}\left[\frac{\partial V}{\partial t}+\left\langle\frac{\partial V}{\partial x}, f(t, x, u)\right\rangle\right] \mathrm{d} t
$$

Because

$$
\varphi(t, x, u)=-\frac{\partial V}{\partial t}-\left\langle\frac{\partial V}{\partial x}, f(t, x, u)\right\rangle
$$

impilies that

$$
\begin{gathered}
y(\beta)-y(\alpha)=-\int_{\alpha}^{\beta} \varphi(t, x, u) \mathrm{d} t, \forall u \in U \\
V(\beta, x(\beta))-V(\alpha, x(\alpha)) \geq-\int_{\alpha}^{\beta} \varphi(t, x, u) \mathrm{d} t \\
V\left(0, x_{0}\right) \leq \int_{0}^{T} \varphi(t, x, u) \mathrm{d} t+V(T, x(T)) \\
V\left(0, x_{0}\right) \leq \int_{0}^{T} \varphi(t, x, u) \mathrm{d} t+h(T, x(T))=I(u)
\end{gathered}
$$

Theorem 3.5. Assume that OCP (3.8) has $V(t, x)$ and there exists feedback $u(t)=g(t, x(t))$ such that:

$$
\begin{aligned}
& \inf _{u \in U}\left[\varphi(t, x, u)+\left\langle\frac{\partial V}{\partial x}, f(t, x, u)\right\rangle\right] \\
& =\varphi\left(t, x^{*}, g\left(t, x^{*}(t)\right)\right)+\left\langle\frac{\partial V}{\partial x}, f\left(t, x^{*}, g\left(t, x^{*}(t)\right)\right)\right\rangle
\end{aligned}
$$

then exists optimal control $u^{*}(t)=g\left(t, x^{*}(t)\right)$ for OCP (3.8).

Proof. Assume that $x^{*}(t)$-one of solutions of control systems (3.1) such that $x^{*}(0)=x_{0} \in H_{0}$ there exists feedback $u^{*}(t)=g\left(t, x^{*}(t)\right)$ :

$$
\begin{aligned}
& \inf _{u \in U}\left[\varphi\left(t, x^{*}(t), u\right)+\left\langle\frac{\partial V}{\partial x}, f\left(t, x^{*}(t), u\right)\right\rangle\right] \\
& =\varphi\left(t, x^{*}(t), g\left(t, x^{*}(t)\right)\right)+\left\langle\frac{\partial V}{\partial x}, f\left(t, x^{*}(t), g\left(t, x^{*}(t)\right)\right)\right\rangle \\
& V(T, x(T))-V(0, x(0))=-\int_{0}^{T} \varphi\left(t, x^{*}(t), g\left(t, x^{*}(t)\right)\right) \mathrm{d} t
\end{aligned}
$$

By lemma 3.3 we have

$$
\begin{aligned}
V(0, x(0))= & h(X(T)) \\
& +\int_{0}^{T} \varphi\left(t, x^{*}(t), g\left(t, x^{*}(t)\right)\right) \mathrm{d} t
\end{aligned}
$$

such that $u^{*}(t)=g\left(t, x^{*}(t)\right)$-optimal control for OCP (3.8).

Definition 3.5. We say that for control system (3.1) are given SOCP - the sheaf-optimization control problem if it denotes:

$$
\left\{\begin{array}{l}
\quad \min _{\substack{u \in U\\
}}(u) \\
\frac{\mathrm{d} x(t)}{\mathrm{d} t}=f(t, x(t), u(t)) \\
H_{t, u}= \\
\left\{x\left(t_{0}, x_{0}, t, u(t)\right) \mid x_{0} \in H_{0} \subset R^{n}, u \in U, t \in[0, T] \subset R^{+}\right\} \\
I(u)=\int_{0}^{T} \iint_{H_{t, u}} \varphi(t, x(t), u(t)) \mathrm{d} x \mathrm{~d} t+\iint_{H_{t, u}} h(x(T)) \mathrm{d} x(T)
\end{array}\right.
$$

where $\iint_{H_{t, u}}$ —integral on $H_{t, u} \subset R^{n}$ and $h(x(T))=V(T, x)$ such that $V(T, x)$ is solution to (HJB) - partial differential equation:

$$
\frac{\partial V}{\partial t}+\frac{\partial V}{\partial x} f+V(t, x) \operatorname{divf}+\varphi(t, x, u)=0
$$

Lemma 3.2. Assume that $V(t, x)$ is a solution of HJB partial differential Equation (3.10) with the boundary conditions:

$$
V(T, x)=h(x(T)), V(0, x)=V\left(0, x_{0}\right)
$$

If function

$W(t, x, u)=\frac{\partial V}{\partial t}+\frac{\partial V}{\partial x} \cdot f+V(t, x) \cdot \operatorname{div} f+\varphi(t, x, u) \geq 0$

and $u(t)$ is admissible control then for optimization control problem SOCP (3.10) there exists estimate:

$$
I(u)-\iint_{H_{0}} V\left(0, x_{0}\right) \mathrm{d} x_{0}=\int_{0}^{T} \int_{H_{t, u}} W(t, x(t), u(t)) \mathrm{d} x \mathrm{~d} t
$$

\section{Proof. Putting}

$$
P(t, u)=\iint_{H_{t, u}} V(t, x) \mathrm{d} x+\int_{0}^{t} \iint_{H_{t, u}} \varphi(t, x(t), u(t)) \mathrm{d} x . \mathrm{d} t(*)
$$

we have:

$$
\begin{aligned}
\frac{\mathrm{d}}{\mathrm{d} t} P(t, u)= & \iint_{H_{t, u}}\left[\frac{\mathrm{d}}{\mathrm{d} t} V(t, x) \mathrm{d} x+V(t, x) \frac{\mathrm{d}}{\mathrm{d} t} \mathrm{~d} x\right] \\
& +\iint_{H_{t, u}} \varphi(t, x(t), u(t)) \mathrm{d} x
\end{aligned}
$$




$$
\begin{aligned}
\frac{\mathrm{d}}{\mathrm{d} t} P(t, u) & =\iint_{H_{t u}}\left[\frac{\partial V}{\partial t}+\frac{\partial V}{\partial x} f+V \cdot \operatorname{divf}+\varphi(t, x(t), u(t))\right] \mathrm{d} x \\
& =\iint_{H_{t, u}} W((t, x(t)), u(t, x(t))) \mathrm{d} x,
\end{aligned}
$$

where $\operatorname{divf} \cdot \mathrm{d} x=\left[\frac{\mathrm{d}}{\mathrm{d} t}(\mathrm{~d} x)\right]$

then

$$
\begin{aligned}
& P(T, u)-P(0, u) \\
& =\int_{0}^{T} \frac{\mathrm{d} P}{\mathrm{~d} t} \mathrm{~d} t=\int_{0}^{T} \iint_{H_{t, u}} W(t, x(t), u(t)) \mathrm{d} x \mathrm{~d} t(* *)
\end{aligned}
$$

By $(*)$ we have

$$
\begin{aligned}
P(T, u) & \left.=\iint_{H_{T, u}} V(T, x) \mathrm{d} x(T)\right)+\int_{0}^{T} \iint_{H_{t, u}} \varphi(t, x, u) \mathrm{d} x \mathrm{~d} t \\
& =\iint_{H_{T, u}} h(x(T)) \mathrm{d} x(T)+\int_{0}^{T} \iint_{H_{t, u}} \varphi(t, x, u) \mathrm{d} x \mathrm{~d} t \\
& =I(u)
\end{aligned}
$$

and $P(0, u)=\iint_{H_{0}} V\left(0, x_{0}\right) \mathrm{d} x_{0}$ then $(* *)$ impilies that

$$
I(u)-\iint_{H_{0}} V\left(0, x_{0}\right) \mathrm{d} x_{0}=\int_{0}^{T} \iint_{H_{t, u}} W(t, x(t), u(t)) \mathrm{d} x \mathrm{~d} t
$$

Theorem 3.6. (Necessary Conditions)

Assume that SOCP (3.10) has solution, that means there exists optimal control $u^{*}(t)$ such that $I\left(u^{*}\right)=\min _{u \in U} I(u)$ and $V(T, x)$ is a solution of HJBpartial differential Equation (3.11) then the necessary conditions for this SOCP (3.10) are:

1) $V(T, x)=h(x(T))$

2) $W\left(t, x^{*}, u^{*}\right)=0$, where

$$
\begin{aligned}
W(t, x, u)= & \frac{\partial V}{\partial t}+\frac{\partial V}{\partial x} f(t, x, u) \\
& +V \cdot \operatorname{divf}(t, x, u)+\varphi(t, x, u)
\end{aligned}
$$

Proof. Suppose that a function SOCP (3.10) that means $I\left(u^{*}\right)=\min _{u \in U} I(u)$. Because $V(t, x)$-solution of HJBpartial differential Equation (3.11):

$$
\frac{\partial V}{\partial t}+\frac{\partial V}{\partial x} f(t, x, u)+V \cdot \operatorname{divf}(t, x, u)+\varphi(t, x, u)=0
$$

with $\frac{\partial V}{\partial t}+\frac{\partial V}{\partial x} f(t, x, u)+V(T, x)=h(x(T))$,

if function $W(t, x, u)$ satisfies:

$$
\begin{aligned}
W(t, x, u)= & \frac{\partial V}{\partial t}+\frac{\partial V}{\partial x} f(t, x, u) \\
& +V \cdot \operatorname{divf}(t, x, u)+\varphi(t, x, u) \geq 0
\end{aligned}
$$

that integrable on sheaf solutions $H_{t, u}$.

By lemma 3.2, if $u(t)$ is admissible control then for optimization control problem SOCP (3.10) there exists estimate:

$$
I(u)-\iint_{H_{0}} V\left(0, x_{0}\right) \mathrm{d} x_{0}=\int_{0}^{T} \iint_{H_{t, u}} W(t, x(t), u(t)) \mathrm{d} x \mathrm{~d} t
$$

Assume that for SOCP (3.10) has optimal control $u^{*}(t)$ then for all $t \in[0, T]$, we have $W\left(t, x^{*}, u^{*}\right)=0$

Theorem 3.7. (Sufficient Conditions)

Assume that $u(t)$ any admissible control for SOCP (3.10) and $V(T, x)$ is a solution of HJB-partial differential Equation (3.11) then the sufficient conditions for this SOCP (3.10) are:

$$
\text { 1) } \begin{aligned}
& V(T, x)= h(x(T)) \\
& \text { 2) } \begin{aligned}
W(t, x, u)= & \frac{\partial V}{\partial t}+\frac{\partial V}{\partial x} f(t, x, u) \\
& +V \cdot \operatorname{divf}(t, x, u)+\varphi(t, x, u) \geq 0
\end{aligned}
\end{aligned}
$$

3) there exists $u^{*}(t)$ such that

$$
I\left(u^{*}\right)=\iint_{H_{0}} V\left(0, x_{0}\right) \mathrm{d} x_{0}
$$

Proof. There exists the other admissible control $\bar{u}(t) \in U \subset R^{d}$, such that for SOCP (3.10) we have

$$
I(\bar{u})-I(u)=\int_{0}^{T} \iint_{H_{t, u}} W(t, \bar{x}, \bar{u}) \mathrm{d} \bar{x} \mathrm{~d} t
$$

By condition (1) of theorem 3.6 we have a function

$$
\begin{aligned}
W(t, x, u)= & \frac{\partial V}{\partial t}+\frac{\partial V}{\partial x} f(t, x, u) \\
& +V \cdot \operatorname{divf}(t, x, u)+\varphi(t, x, u)
\end{aligned}
$$

that integrable on sheaf solutions $H_{t, u}$.

$$
I(u)=\int_{0}^{T} \iint_{H_{t, u}} \varphi(t, x(t), u(t)) \mathrm{d} x \mathrm{~d} t+\iint_{H_{t, u}} h(x(T)) \mathrm{d} x(T)
$$

We find the function $V(t, x)$ from equation:

$$
\frac{\partial V}{\partial t}+\frac{\partial V}{\partial x} f(t, x, u)+V \cdot \operatorname{div} f(t, x, u)+\varphi(t, x, u)=0
$$

with condition $V(T, x)=h(x(T))$.

By condition (2) of this theorem:

$$
I\left(u^{*}\right)-\iint_{H_{0}} V\left(0, x_{0}\right) \mathrm{d} x_{0}=\int_{0}^{T} \iint_{H_{t, u}} W\left(t, x^{*}(t), u^{*}(t)\right) \mathrm{d} x \mathrm{~d} t=0
$$

and implies that $u^{*}(t)=g\left(t, x^{*}(t)\right)$ —optimal control for SOCP (3.10).

Example 3.1. When using missiles not for the purpose of shooting down aircraft noise bomb attack as B52 shot if only 01 or 02 rockets can not succeed. The rockets theit fire it will be the interference or escort aircraft will 
be explosive.

A problem arises: What to do in order to shoot down aircraft noise when operating in the sky. To solve this problem, we must fire simultaneously from SAM sites from 03 or more results. The rockets have to be controlled from headquarters and shot to pick the exact point-B52.

Mathematical model for problem shooting attack aircraft noise control system with (3.10), the test bundle (2.4) and optimization problem are (SOCP) in the above with $n=3$ (see Figure 2).

\section{Conclusions}

The Sheaf Optimization problem for Control Systems

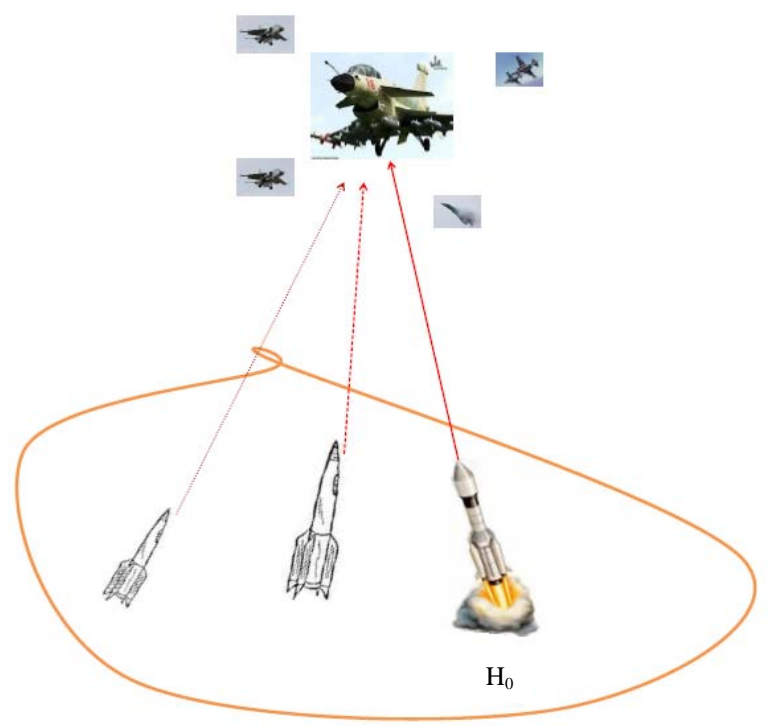

Figure 2. The sheaf of SAM shooting down aircraft noise bomb attack as B52.
(SOPCS) have a high practical significance, as the series of SAM to destroy B52 attack aircraft with fighter jamming, or laser beam to destroy targets, like the beams in materials research of Physical nuclear, etc, ... This paper described some types of sheaf optimal problems. We can solve them by Pontryagin's Principle, Lyapunov's Energy Function or by the Hamilton's Principle. In this paper we present the necessary and sufficient conditions for this problem by Hamilton's Principle, namely by HJB equations.

In the near future, we will set the numerical calculations can be applied to a clearer and will study the different Optimization problems with some controls $u(t), v(t)$.

\section{Acknowledgements}

The authors gratefully acknowledge the referees for their careful reading and many valuable remarks which improved the presentation of the paper.

\section{REFERENCES}

[1] A. D. Ovsyannikov, "Mathematical Methods in Sheaf Controls,” Leningrad University Pub., Saint Petersburg, 1980.

[2] N. D. Phu and T. T. Tung, "Some Properties of SheafSolutions of Sheaf Fuzzy Control Problems," Electronic Journal of Differential Equations, Vol. 2006, No. 108, 2006, pp. 1-8.

[3] N. D. Phu and T. T. Tung, "Some Results on Sheaf Solutions of Sheaf Set Control Differential Equations," Journal of Nonlinear Analysis: Theory, Methods \& Applications, Vol. 67, No. 5, 2007, pp. 1309-1315. doi:10.1016/j.na.2006.07.018

[4] N. D. Phu, "On the Global Controllable for Set Control Differential Equations," International Journal of Evolution Equations, Vol. 4, No. 3, 2009, pp. 281-292. 